\title{
Glaucome et vasoconstricteurs
}

\author{
Note de la rédaction \\ A la suite de la parution des recommandations sur l'emploi des vasoconstricteurs en \\ odonto-stomatologie publiées par la SFMBCB (Med Buccal Chir Buccal 2003;9:65-94), \\ il a été signalé qu'une contre-indication importante à l'utilisation des vasoconstricteurs, \\ le glaucome, n'était pas citée dans ces recommandations. \\ Voici la réponse transmise par le Dr Carlos MADRID, rapporteur du groupe de travail \\ sur l'emploi des vasoconstricteurs en odonto-stomatologie.
}

II convient tout d'abord de restreindre le cadre de cette réponse à deux des 4 formes de glaucome qui sont à notre sens et de très loin les plus fréquentes - le glaucome à angle ouvert et le glaucome à angle fermé - et qui sont en outre les seules formes pour lesquelles la physiopathologie puisse faire envisager un effet hémodynamique délétère potentiel des vasoconstricteurs odonto-stomatologiques.

Le glaucome à angle ouvert atteint $0,5 \%$ de la population générale aux USA et en Europe. II traduit une gêne à l'écoulement de l'humeur aqueuse à travers le trabéculum c'est à dire dans l'angle formé entre la cornée et l'iris. La pression intra-oculaire est alors supérieure à $21 \mathrm{~mm}$ de mercure. II s'y associe une destruction progressive des fibres axoniques du nerf optique et des modifications par excavation de la papille.

Les formes chroniques sont le plus souvent asymptomatiques et l'hypertonie oculaire qui précède le plus souvent n'est traitée qu'à partir d'une pression de $30 \mathrm{~mm}$ de $\mathrm{Hg}$ et ne dégénère pas systématiquement en glaucome puisque seules $10 \%$ des hypertonies deviennent des glaucomes par décennie.

Quel en est le traitement ? II est pour l'essentiel médical : il fait appel à l'administration de collyres parasympathomimétiques (pilocarpine), de béta bloquants comme le timolol, et... de sympathomimétiques comme l'épinephrine ou l'adré- naline. Ces derniers permettent de réduire la pression intra-oculaire en diminuant la production de l'humeur aqueuse et en augmentant son écoulement.

On conçoit donc que si l'on peut utiliser seule ou plus fréquemment en association avec un collyre atropinique (Glaucadrine $\AA$ ), l'adrénaline topique (0,1\% dans l'étude de Groh et coll, $1999 ; 0,1 \%$ de dipivéphrine dans Propine $₫$ commercialisée en France) dans le traitement du glaucome à angle ouvert, les risques oculaires des effets systémiques de la résorption d'un anesthésique local avec 1:200 000 d'adrénaline sont probablement en dessous de ce qui est mesurable et il ne l'ont d'ailleurs pas été puisqu'aucune publication expérimentale ou épidémiologique sur ce thème n'a été retrouvée ni par notre recherche bibliographique large rapportée dans la méthodologie de notre travail ni par la recherche bibliographique ciblée sur le glaucome que j'ai réalisée après votre question.

Le glaucome à angle fermé ne représente aux USA, par exemple, que $10 \%$ des patients atteints de la forme à angle ouvert. C'est avant tout un tableau d'urgence thérapeutique qui ne survient que sur des yeux anatomiquement prédisposés. Il se déclare le plus souvent au cours d'une dilatation pupillaire thérapeutique, c'est à dire très loin du contexte du cabinet dentaire. Le glaucome à angle fermé est traité le plus sou- médecine

buccale

chirurgie

buccale

VOL. $9, \mathrm{~N}^{\circ} 3$ 2003

page 147 
médecine

buccale

chirurgie

buccale

VOL. $9, \mathrm{~N}^{\circ} 3$ 2003

page 148 vent chirurgicalement après la normalisation médicale de la tension oculaire. L'établissement chirurgical d'une communication entre les chambres antérieure et postérieure de l'œil à travers l'iris, ramène la pression oculaire dans les limites de l'hypertonie oculaire.

L'absence du glaucome dans notre revue des affections qui " contre-indiqueraient " l'emploi des vasoconstricteurs en odonto-stomatologie, n'est donc pas un « oubli » mais découle de l'absence de cette contre-indication dans la littérature qui fait autorité en la matière. Rood et Meechan (1997), Budentz (2000), Gaudy et Aretto (1999), Gay et Berini (1997) n'en font aucune mention dans les différentes revues de contre-indications qu'ils publient.

Dans la série de Daublander et coll (1997), les complications liées à l'anesthésie locale odonto-stomatologique sont répertoriées chez 2731 patients: aucune complication glaucomateuse n'est rapportée alors même que $18,4 \%$ de ces patients présentaient un facteur de risque hypertensif traité par béta bloquant ou inhibiteur calcique et que l'hypertension artérielle est un facteur de risque reconnu pour le glaucome à angle ouvert (Hennis et coll, 2003).

En tentant de comprendre ce qui peut faire penser que le glaucome à angle ouvert est une contre-indication des vasoconstricteurs en anesthésie odonto-stomatologique je me suis souvenu que le Vidal ${ }^{\circledR}$ mentionne encore cette contre-indi- cation dans les RCP de l'articaine associée à l'adrénaline et de la lidocaïne associée à la noradrénaline ou à l'adrénaline. Nous avons dit ce que nous pensions de la nor-adrénaline dans notre rapport et de ses effets vasculaires périphériques potentiellement plus sévères que ceux de l'adrénaline. Mais nous pensons que le risque de déclencher un glaucome à angle fermé lors de l'utilisation de dosages normaux d'anesthésiques locaux adrénalinés en odonto-stomatologie est un risque théorique potentiel qui n'a été ni évalué ni démontré. L'absence de cas clinique clairement rapporté à cette étiopathogénie iatrogène et correctement documenté confirme que la mention du Vidal tient plus du principe de précaution médico-légale que de la réalité scientifique. J'observe avec satisfaction que le Dorosz $₫ 2002$ et 2003 ne reprend pas cette contre-indication dans la présentation des formes adrénalinées (1:100 000 et 1:200 000) des anesthésiques locaux injectables (lidocaïne, bupivacaïne).

Ces recommandations avaient pour but, entre autres de tordre le cou à un certain nombre de mythes médicaux au sujet des vasoconstricteurs. Nous n'avions pas cru nécessaire de faire preuve d'acharnement sur celui du glaucome qui nous paraissait trépassé. Cette remarque nous rappelle utilement à l'exhaustivité.

\section{Carlos MADRID}

Rapporteur du Groupe de Travail CHU Toulouse Hôtel Dieu 